\title{
THE SPECTROSCOPIC CLASSIFICATION AND EXPLOSION PROPERTIES OF SN 2009nz ASSOCIATED WITH GRB 091127 AT $z=0.490$
}

\author{
E. Berger ${ }^{1}$, R. Chornock ${ }^{1}$, T. R. Holmes ${ }^{1}$, R. J. Foley ${ }^{1,7}$, A. Cucchiara ${ }^{2,3}$, C. Wolf $^{4}$, \\ PH. Podsiadlowski ${ }^{4}$, D. B. Fox ${ }^{5}$, AND K. C. Roth ${ }^{6}$ \\ ${ }^{1}$ Harvard-Smithsonian Center for Astrophysics, 60 Garden Street, Cambridge, MA 02138, USA \\ ${ }^{2}$ Lawrence Berkeley National Laboratory, M.S. 50-F, 1 Cyclotron Road, Berkeley, CA 94720, USA \\ ${ }^{3}$ Department of Astronomy, 601 Campbell Hall, University of California, Berkeley, CA 94720-3411, USA \\ ${ }^{4}$ Department of Astrophysics, Denys Wilkinson Building, University of Oxford, Keble Road, Oxford OX1 3RH, UK \\ ${ }^{5}$ Department of Astronomy and Astrophysics, Pennsylvania State University, 525 Davey Laboratory, University Park, PA 16802, USA \\ ${ }^{6}$ Gemini Observatory, 670 N. Aohoku Place Hilo, HI 96720, USA \\ Received 2011 June 15; accepted 2011 September 7; published 2011 December 6
}

\begin{abstract}
We present spectroscopic observations of GRB $091127(z=0.490)$ at the peak of the putative associated supernova SN 2009nz. Subtracting a late-time spectrum of the host galaxy, we isolate the contribution of SN 2009nz and uncover broad features typical of nearby gamma-ray-burst-supernovae (GRB-SNe). This establishes unambiguously that GRB 091127 was accompanied by a broad-lined Type Ic SN, and links a cosmological long burst with a standard energy release $\left(E_{\gamma, \text { iso }} \approx 1.1 \times 10^{52} \mathrm{erg}\right.$ ) to a massive star progenitor. The spectrum of SN $2009 \mathrm{nz}$ closely resembles that of SN 2006aj, with SN 2003dh also providing an acceptable match, but has significantly narrower features than SNe 1998bw and 2010bh, indicative of a lower expansion velocity. The photospheric velocity inferred from the Si II $\lambda 6355$ absorption feature, $v_{\mathrm{ph}} \approx 17,000 \mathrm{~km} \mathrm{~s}^{-1}$, is indeed closer to that of SNe 2006aj and 2003dh than to the other GRB-SNe. Combining the measured velocity with the light curve peak brightness and width, we estimate the following nominal (maximal) explosion parameters: $M_{\mathrm{Ni}} \approx 0.35(0.6) M_{\odot}, E_{K} \approx 2.3 \times 10^{51}\left(8.4 \times 10^{51}\right) \mathrm{erg}$, and $M_{\mathrm{ej}} \approx 1.4$ (3.5) $M_{\odot}$, similar to those of SN 2006aj. These properties indicate that SN 2009nz follows a trend of lower $M_{\mathrm{Ni}}$ for GRB-SNe with lower $E_{K}$ and $M_{\mathrm{ej}}$. Equally important, since GRB 091127 is a typical cosmological burst, the similarity of SN 2009nz to SN 2006aj either casts doubt on the claim that XRF 060218/SN 2006aj was powered by a neutron star or indicates that the nature of the central engine is encoded in the SN properties but not in the prompt emission. Future spectra of GRB-SNe at $z \gtrsim 0.3$ will shed light on the full dispersion of SN properties for standard long GRBs, on the relation between SNe associated with sub-energetic and standard GRBs, and on a potential dispersion in the associated SN types.
\end{abstract}

Key words: gamma-ray burst: general - gamma-ray burst: individual (GRB 091127)

Online-only material: color figures

\section{INTRODUCTION}

The association of long-duration gamma-ray bursts (GRBs) with Type Ic supernovae ( $\mathrm{SNe}$ ) provides the most direct evidence for massive stripped-envelope (Wolf-Rayet) stars as the progenitors of long GRBs (e.g., Woosley \& Bloom 2006). This connection was first intimated by the discovery of the unusually luminous and broad-lined Type Ic SN 1998bw $(d=40 \mathrm{Mpc})$ in spatial and temporal coincidence with GRB 980425 (Galama et al. 1998). It was further supported by the detection of late-time photometric "bumps" in the optical light curves of several GRBs at $z \lesssim 1$ that resembled the optical light curve of SN 1998bw (e.g., Bloom et al. 1999; Lazzati et al. 2001; Bloom et al. 2002; Soderberg et al. 2005, 2006b), although alternative explanations for these bumps have been proposed (Esin \& Blandford 2000; Waxman \& Draine 2000). Subsequently, unambiguous spectroscopic identifications were obtained for four nearby GRBs: GRB 030329/SN 2003dh $(z=0.169$; Hjorth et al. 2003; Stanek et al. 2003), GRB 031203/SN 20031w ( $z=0.105$; Malesani et al. 2004), XRF 060218/SN 2006aj $(z=0.033$; Modjaz et al. 2006; Pian et al. 2006), and GRB 100316D/SN 2010bh $(z=0.059$; Chornock et al. 2010). All four events are broad-lined Type Ic SNe, similar to SN 1998bw. However, only GRB 030329 is typical of the cosmological long GRB

\footnotetext{
7 Clay Fellow.
}

population in terms of its energy scale (Berger et al. 2003), while the remaining three events are all sub-energetic in $\gamma$-rays, have no clear optical afterglows, exhibit only mildly relativistic velocities, and appear to be quasi-spherical (the so-called sub-energetic GRBs; Soderberg et al. 2004, 2006a).

As a result, there is a clear impetus to obtain spectroscopic observations of GRB-SNe at $z \gtrsim 0.3$ to bolster the GRB-SN connection for the cosmological population, as well as to map the range of GRB-SN properties (i.e., velocity, kinetic energy, ejecta mass, ${ }^{56} \mathrm{Ni}$ mass), and to compare the properties of the $\mathrm{SNe}$ associated with standard and sub-energetic GRBs. The challenge of obtaining such spectroscopic observations is twofold. First, the associated SNe are faint, with a peak optical apparent brightness ${ }^{8}$ of $i_{\mathrm{AB}} \approx 22.5$ mag at $z \sim 0.5$ and $\approx 24.5$ mag at $z \sim 1$. Second, at these redshifts the brightness of the $\mathrm{SNe}$ and the compact host galaxies ${ }^{9}$ are similar, therefore requiring a careful subtraction of the host spectrum to robustly reveal and properly measure the $\mathrm{SN}$ features.

To date, spectroscopic observations timed to coincide with potential GRB-SNe at $z \sim 0.3-1$ have been obtained in five cases. Della Valle et al. (2003) obtained a spectrum of GRB 021211

\footnotetext{
8 This is due in part to line-blanketing in the rest-frame UV, which is redshifted into the optical band at $z \gtrsim 0.5$.

9 The typical half-light radii of long GRB host galaxies at $z \gtrsim 0.3$ are $\lesssim 0$ '.5 (Bloom et al. 2002; Wainwright et al. 2007).
} 
$(z=1.006)$ during the peak of an associated bump (designated SN 2002lt). The putative SN and host galaxy had a comparable brightness of $R \approx 25.2 \mathrm{mag}$, but the host contribution was not subtracted from the spectrum, which appears to exhibit a broad minimum interpreted as $\mathrm{Ca}$ II $\mathrm{H} \& \mathrm{~K}$ blueshifted by about $15,000 \mathrm{~km} \mathrm{~s}^{-1}$. Della Valle et al. (2003) claim a close match with the normal Type Ic SN 1994I, but a poor match with SN 1998bw; however, inspection of their Figure 2 shows that the peculiar Type Ia SN $1991 \mathrm{bg}$ is an equally good match to the noisy spectrum of the putative SN 20021t. Garnavich et al. (2003) obtained a spectrum of GRB $011121(z=0.362)$ coincident with the peak of an associated bump (designated SN 2001ke) when the optical brightness ${ }^{10}$ was $R \approx 23$ mag. Unlike SN 1998bw, the resulting spectrum is flat at $\lambda_{\text {rest }} \lesssim 5100 \AA$, which Garnavich et al. (2003) claim as evidence for a Type IIn event. However, the large, and uncertain, extinction along this line of sight may influence this result. A spectrum of XRF $020903(z=0.251)$ was obtained by Soderberg et al. (2005) during a late flattening in the light curve. After subtraction of a model starburst galaxy template, the residual spectrum exhibits an overall shape similar to SN 1998bw. Della Valle et al. (2006) obtained a spectrum of GRB 050525A ( $z=0.606)$ about two weeks after the peak of the associated bump (designated SN 2005nc), with equal contribution from the putative SN and host galaxy (each with $R \approx 25.2 \mathrm{mag}$ ). After subtraction of a model host spectrum, the residual spectrum exhibits a broad bump at $\lambda_{\text {rest }} \approx 5100 \AA$, with a decline at longer wavelengths, broadly resembling the spectrum of SN 1998bw. Sparre et al. (2011) obtained spectra of GRB 101219B ( $z=0.552$ based on weak Mg II absorption) about 16 and 37 days after the burst and find broad undulations that resemble SN 1998bw and distinct from an earlier featureless spectrum. They do not directly account for the spectral shape of the host galaxy, assuming instead a flat spectrum. In none of the five cases was an attempt made to extract any SN properties beyond addressing whether or not they resembled SN 1998bw.

Here we present spectroscopic observations of GRB 091127 at $z=0.490$, timed to coinciding with the peak of a photometric bump designated SN 2009nz (Cobb et al. 2010). The bump light curve resembles SN 1998bw, albeit dimmer by about 0.1 mag (within the uncertainty of both light curves) and with a somewhat earlier peak and narrower light curve; it is brighter than SN 2006aj by about $0.25 \mathrm{mag}$, with a later peak and broader light curve (Cobb et al. 2010). At the peak of the bump, the $\mathrm{SN}$ brightness is $I \approx 22.3 \mathrm{mag}$, the afterglow is estimated to be $I \approx 24.2 \mathrm{mag}$, and the host galaxy has $I \approx 22.5 \mathrm{mag}$ (i.e., the relative contributions are about $50 \%, 8 \%$, and $42 \%$, respectively). Thus, as in the previous cases of $z \gtrsim 0.3$ bumps, the host and putative supernovae contribute a similar amount of flux, thereby requiring a careful subtraction of the host galaxy spectrum. Here we undertake this procedure using a spectrum of the host galaxy obtained about 1 year after the burst (Section 2). Subtraction of the host contribution cleanly isolates the SN spectrum and reveals broad undulations that clearly resemble previous GRB-SNe, in particular SN 2006aj and potentially SN 2003dh, but with narrower features than SN 1998bw and SN 2010bh (Section 3). This result demonstrates that the photometric bump is indeed an SN and allows us to extract the explosion properties of SN 2009nz (Section 3). We compare these properties to the well-studied nearby GRB-SNe to explore

\footnotetext{
10 It is unclear from Garnavich et al. (2003) what fraction of the flux within the $1^{\prime \prime}$ slit is contributed by the extended host galaxy. The host contribution was not subtracted from the spectrum.
}

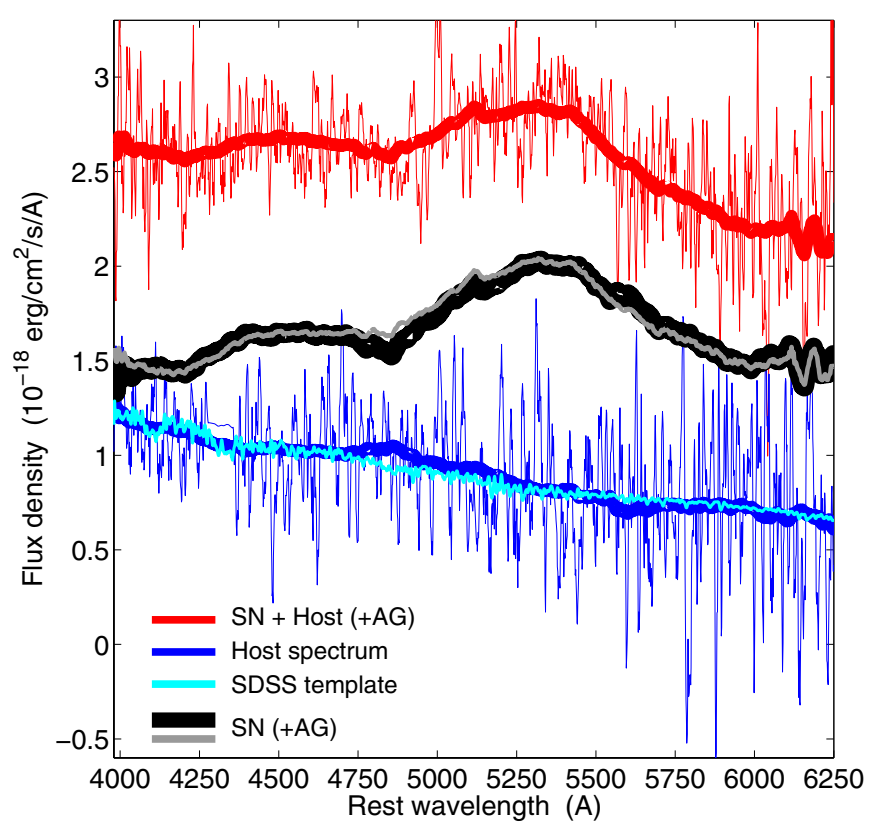

Figure 1. Spectra of the GRB 091127/SN 2009nz "bump" (red) and of the host galaxy (blue). The thick lines are smoothed with a $100 \AA$ A boxcar. The subtracted spectrum (with about $15 \%$ contribution from the featureless afterglow; Cobb et al. 2010) is shown as a thick black line. The undulations typical of a broad-lined GRB-SN are clearly seen, with prominent absorption features at $\lambda_{\text {rest }} \approx 4200,4750$, and $6000 \AA$. We also perform a subtraction relative to a template blue galaxy spectrum from the SDSS (cyan, emission lines clipped) and find that the resulting SN spectrum (gray) is nearly identical to the spectrum from actual host subtraction.

(A color version of this figure is available in the online journal.)

the dispersion of SN properties for standard and sub-energetic GRBs (Section 4).

\section{SPECTROSCOPIC OBSERVATIONS}

\section{1. “Bump” Spectrum}

We observed GRB $091127(z=0.490$; Cucchiara et al. 2009) with the Gemini Multi-Object Spectrograph (GMOS) on the Gemini-North $8 \mathrm{~m}$ telescope (program GN-2009B-Q28, PI: Fox) on 2009 December 22.27 UT. The spectrum was timed to coincide with the peak of an associated SN 1998bwlike event at $z=0.490$. Two dithered $900 \mathrm{~s}$ exposure pairs were obtained with the R400 grating at central wavelengths of 8000 and $8050 \AA$ in 0 '.65 seeing. The resulting combined spectrum covers 5900-9500 $\AA$ at a resolution of about $7 \AA$. An OG515 order-blocking filter was used to prevent second-order contamination in the red part of the spectrum.

The spectra were reduced, combined, and extracted using standard procedures in IRAF, while flux calibration and correction for telluric absorption were performed using custom IDL routines with archival observations of the standard stars BD+28 4211 and Feige 34, respectively. The BD+28 $4211 \mathrm{spec}-$ trum was obtained 3.5 months prior to our observations and was used to obtain the correct spectral shape, with the overall flux calibration scaled by photometry (see below). The telluric correction does not influence the spectrum significantly since the dominant telluric features $(\approx 6870$ and $7610 \AA$ ) do not coincide with any intrinsic spectral features (Figure 1). The mean air mass of the observation was 1.3 and the $1^{\prime \prime}$ slit was aligned at the parallactic angle, so the relative spectral shape is reliable. We determine the overall flux scale of the spectrum by 
Table 1

Magellan/IMACS Host Galaxy Photometry

\begin{tabular}{lccc}
\hline \hline Filter & $\begin{array}{c}\text { Exposure Time } \\
(\mathrm{s})\end{array}$ & $\begin{array}{c}m_{\mathrm{AB}} \\
(\mathrm{mag})\end{array}$ & $\begin{array}{c}F_{v}{ }^{\mathrm{a}} \\
(\mu \mathrm{Jy})\end{array}$ \\
\hline$g$ & 420 & $24.05 \pm 0.14$ & $0.99 \pm 0.14$ \\
$r$ & 720 & $23.34 \pm 0.05$ & $1.84 \pm 0.09$ \\
$i$ & 480 & $22.89 \pm 0.07$ & $2.70 \pm 0.19$ \\
$z$ & 720 & $22.57 \pm 0.10$ & $3.56 \pm 0.36$ \\
\hline
\end{tabular}

Note.

${ }^{\text {a }}$ Corrected for Galactic extinction of $E(B-V)=0.038$ mag (Schlegel et al. 1998).

integrating over the $r$-band filter response function and comparing to photometry of the target in the GMOS $r$-band acquisition image within the same aperture size $(r=22.66 \pm 0.06$ mag calibrated relative to follow up observations from Magellan-see Section 2.2). We also verify the calibration by comparison to the value reported by Cobb et al. (2010) of $I \approx 22.3 \mathrm{mag}$. The overall flux calibration is therefore known to better than $10 \%$.

\subsection{Host Galaxy Spectrum}

Spectroscopic and photometric observations of the host galaxy of GRB 091127 were obtained with the Inamori Magellan Areal Camera and Spectrograph (IMACS) mounted on the Magellan/Baade $6.5 \mathrm{~m}$ telescope on the nights of 2010 November 13 and 14 UT. We obtained two 2400 s exposures with the $2001 \mathrm{~mm}^{-1}$ grism using a 0 '.9 slit and a $4950 \AA$ orderblocking filter to avoid second-order contamination in the red part of the spectrum. The spectra were obtained at the parallactic angle, and the seeing during the observations was 0 . $^{\prime} 6$, well matched to the conditions of the GMOS spectrum. The spectra were processed using standard procedures in IRAF, and the wavelength calibration was performed using HeNeAr arc lamps. The resulting wavelength coverage is 5000-9400 $\AA$, with a resolution of about $4.7 \AA$. Flux calibration was performed using observations of the spectrophotometric standard star Feige 110. We confirmed the overall flux calibration and shape by integrating the spectrum over the $r$-and $i$-band filter response functions $(r \approx 23.5 \mathrm{mag}, i \approx 23.3 \mathrm{mag}$ ) compared to photometric measurements of the host within the same aperture size $(r \approx 23.5$ mag, $i \approx 23.2 \mathrm{mag}$ ). As in the case of the "bump" spectrum, the overall flux calibration is known to better than $10 \%$.

The photometric observations were obtained in the griz filters. The data were processed using standard procedures in IRAF, and photometric calibration was performed using observations of the southern standard star field E3A. The resulting flux measurements (in a $1^{\prime \prime} .5$ radius aperture) are provided in Table 1 . We note that our $i$-band measurement is in excellent agreement with the $I$-band host flux given in Cobb et al. (2010).

\section{THE SPECTRUM OF SN 2009nz AND A COMPARISON TO NEARBY GRB-SNe}

The spectra at the peak of the photometric bump and of the host galaxy alone are shown in Figure 1. The bump spectrum (supernova+host) exhibits broad undulations with a peak at $\lambda_{\text {rest }} \approx 5300 \AA$, and a possible second peak at $\lambda_{\text {rest }} \approx 4500 \AA$. These features are reminiscent of previous GRB-SNe, but it is critical to ensure that they do not result from host galaxy contamination, since the host contributes about half of the total flux. To remove any possible contamination, and to cleanly isolate the $\mathrm{SN}$ features, we subtract the host spectrum from the bump spectrum and find that the features are robustly detected. We perform the subtraction by interpolating both spectra onto a common wavelength scale $\left(1 \AA\right.$ pixel $\left.^{-1}\right)$ over the range of wavelengths shared by both spectra. We do not subtract the afterglow contribution since it only accounts for $8 \%$ of the total flux (or about $15 \%$ of the SN flux; Cobb et al. 2010) and its power-law shape would at most tilt the overall SN spectrum without affecting the robustness or location of the spectral features.

The isolated spectrum of the transient exhibits three absorption troughs centered at rest wavelengths of about 4200, 4750, and $6000 \AA$, closely matching the spectra of nearby GRB-SNe. The 4200 and $4750 \AA$ features are generally identified as blends of Fe II/Ti II and Fe II/Fe III, respectively, while the $6000 \AA$ feature is identified as $\mathrm{Si}$ II $\lambda 6355$. We confirmed these results by subtracting a scaled blue galaxy template spectrum obtained from the Sloan Digital Sky Survey cross-correlation library ${ }^{11}$ (Figure 1). The galaxy spectral shape is essentially identical to our measurements, and the resulting isolated SN spectrum reveals the same features detected in the subtraction relative to the Magellan host spectrum. In the analysis below we use the SN spectrum from the actual host subtraction, but note that the results are unchanged for the spectrum from template subtraction.

We compare our SN 2009nz spectrum at $\delta t_{\text {rest }}=16.3$ days (corresponding to $\approx 1.5$ day past maximum light; Cobb et al. 2010) to the spectra of the nearby GRB-SNe 1998bw, 2003dh, 2006aj, and 2010bh on a similar timescale (Figure 2). Overall, the spectrum of SN 2009nz is remarkably similar to the nearby GRB-SNe, exhibiting the same broad features that are a hallmark of large expansion velocities. This unambiguously identifies the photometric bump associated with GRB 091127 as a supernova and demonstrates that SN 2009nz is also a broadlined event, likely ${ }^{12}$ of Type Ic. We do not find a prominent He I $\lambda 5876$ absorption feature as expected for a Type Ib SN. Finally, a comparison to the normal Type Ic SN 1994I and the normal Type Ib SN 1998dt reveals a poor match due to their significantly narrower features.

Among the sample of nearby GRB-SNe the best overall match to SN 2009nz is provided by SN 2006aj, in terms of both the location of the absorption minima and the width of the features (Figure 2). SN 2003dh also provides a reasonable match, but SNe 1998bw and 2010bh have significantly broader features indicative of larger expansion velocities. We estimate the photospheric velocity using the location of the Si II $\lambda 6355$ absorption feature (Figure 2). We find that the for SN 2009nz the minimum is located at $\lambda_{\text {rest }} \approx 6000 \AA$ (with an uncertainty of about $50 \AA$, inferred from fitting the feature with a Gaussian profile and a range of boxcar smoothing parameters). This corresponds to a velocity of $v_{\mathrm{ph}} \approx 17,000 \mathrm{~km} \mathrm{~s}^{-1}$ (with an uncertainty of about $2500 \mathrm{~km} \mathrm{~s}^{-1}$ ). A comparison to the inferred Si II $\lambda 6355$ velocities of the previous GRB-SNe (Chornock et al. 2010) indeed indicates a similar velocity to SN $2006 a j\left(\approx 19,000 \mathrm{~km} \mathrm{~s}^{-1}\right)$, and lower velocity than SN 1998bw $\left(\approx 24,000 \mathrm{~km} \mathrm{~s}^{-1}\right)$ and SN 2010bh ( $\left.\approx 25,000 \mathrm{~km} \mathrm{~s}^{-1}\right)$.

Using the photospheric velocity from our spectrum, along with the peak brightness $\left(M_{V}\right)$ and width of the photometric light curve (parameterized as $\Delta m_{15}$ ), we can determine the basic

\footnotetext{
11 http://www.sdss.org/dr7/algorithms/spectemplates/index.html

12 Although our spectrum does not cover $\mathrm{H} \alpha$, we do not see an obvious feature corresponding to $\mathrm{H} \beta$ at an expected blueshift of $\sim 10,000-30,000 \mathrm{~km} \mathrm{~s}^{-1}$. Furthermore, the overall similarity to the nearby well-studied GRB-SNe, which are all of Type Ic, suggests that SN 2009nz shares this designation.
} 


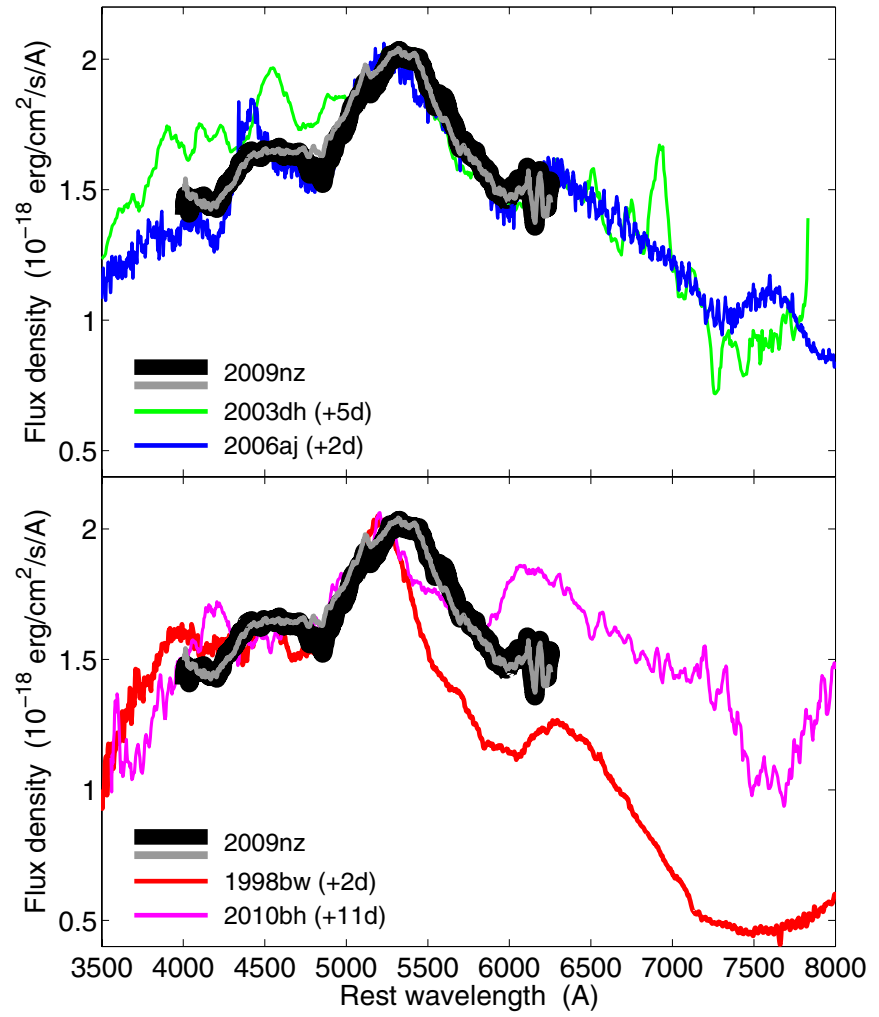

Figure 2. Spectrum of SN 2009nz (black, with about $15 \%$ contribution from the featureless afterglow; Cobb et al. 2010) compared to spectra of well-studied nearby GRB-SNe on a comparable timescale (the times in parentheses are relative to the peak of the light curve). Top: SN 2003dh (Matheson et al. 2003) and SN 2006aj (Modjaz et al. 2006); bottom: SN 1998bw (Patat et al. 2001) and SN 2010bh (Chornock et al. 2010). The comparison spectra have been normalized to the same flux at the peak of the $\lambda_{\text {rest }} \approx 5300 \AA$ feature. The spectrum of SN 2009nz closely resembles the previous GRB-SN, with the best overall match provided by SN 2006aj. SN 2003dh also provides a reasonable match in terms of the width of the features, while SNe 1998bw and 2010bh have broader features indicative of larger expansion velocities. The spectrum from a template galaxy subtraction (gray) is also shown.

(A color version of this figure is available in the online journal.)

explosion properties of SN 2009nz, namely, the kinetic energy $\left(E_{K}\right)$, the ejecta mass $\left(M_{\mathrm{ej}}\right)$, and the mass of synthesized ${ }^{56} \mathrm{Ni}$ $\left(M_{\mathrm{Ni}}\right)$. The light curve presented in Cobb et al. (2010) indicates $M_{V}=-19.0 \pm 0.2 \mathrm{mag}$ and $\Delta m_{15}(V)=1.3 \pm 0.3 \mathrm{mag}$. Using the formulation of Drout et al. (2010), based on the theoretical model of Arnett (1982), we $\operatorname{infer}{ }^{13} M_{\mathrm{Ni}, \odot} \approx 0.35$ and $M_{\mathrm{ej}, \odot}^{3 / 4} / E_{K, 51}^{1 / 4} \approx 1.0$ (here $M_{\mathrm{ej}, \odot}$ is the ejecta mass in units of solar masses and $E_{K, 51}$ is the kinetic energy in units of $10^{51} \mathrm{erg}$ ). From our inferred velocity we also find $E_{K, 51} / M_{\mathrm{ej}, \odot} \approx 1.7$, and therefore $E_{K, 51} \approx 2.3$ and $M_{\mathrm{ej}, \odot} \approx 1.4$. As with the overall shape of the spectrum, the inferred explosions properties of SN 2009nz most closely resemble those of SN 2006aj, for which Mazzali et al. (2006a) estimated $M_{\mathrm{Ni}, \odot} \approx 0.2, E_{K, 51} \approx 2$, and $M_{\mathrm{ej}, \odot} \approx 2$. On the other hand, they are significantly lower than for SNe 1998bw and 20031w; see Table 2 and Figure 3. The maximal values of the explosion properties given the uncertainties in $M_{V}, \Delta m_{15}$, and $v_{\text {ej }}$ are $M_{\mathrm{Ni}, \odot} \approx 0.6$, $E_{K, 51} \approx 8.4$, and $M_{\mathrm{ej}, \odot} \approx 3.5$.

${ }_{13}$ We use a mean difference of $\approx 0.3 \mathrm{mag}$ between $\Delta m_{15}(V)$ and $\Delta m_{15}(R)$, as well as between $M_{V}$ and $M_{R}$, as found by Drout et al. (2010), to place SN 2009nz on their grid of models. The models assume energy deposition from ${ }^{56} \mathrm{Co}$ and ${ }^{56} \mathrm{Ni}$, a homogeneous density distribution of the ejecta, and a fixed optical opacity.

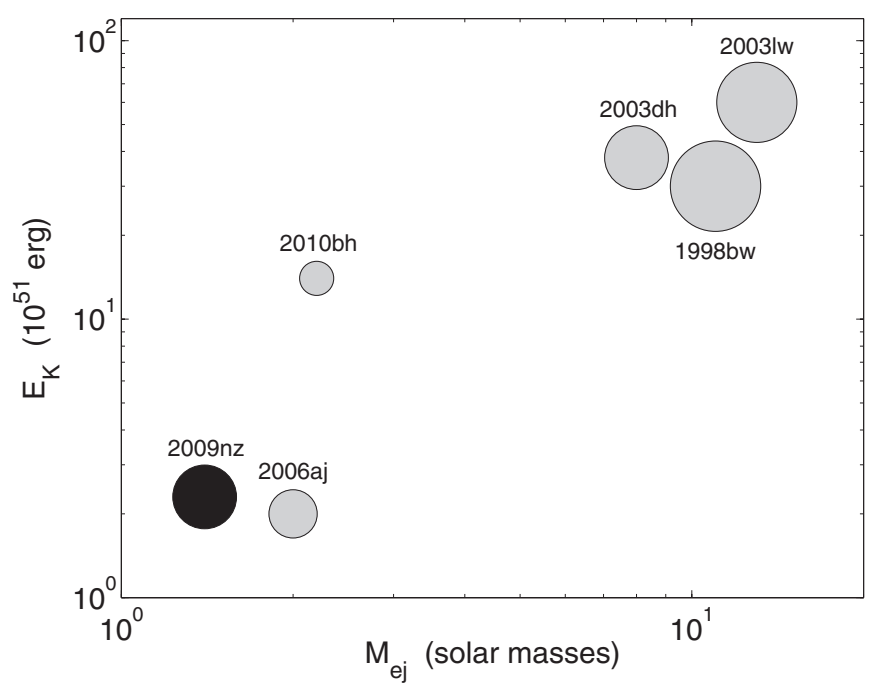

Figure 3. Explosion properties of the well-studied nearby GRB-SNe (gray circles), along with our inferred properties of SN 2009nz (black circle). The area of the symbols is linearly proportional to the mass of synthesized ${ }^{56} \mathrm{Ni}$ for each event. There appears to be a broad correlation between the three explosion properties, with the most energetic GRB-SNe producing the largest ${ }^{56} \mathrm{Ni}$ and ejecta masses. The overall correlation between $E_{K}$ and $M_{\mathrm{ej}}$ reflects the range of ejecta velocities of $\sim 10,000-30,000 \mathrm{~km} \mathrm{~s}^{-1}$. References for the individual events are given in Table 2.

Table 2

Explosion Properties of GRB-SNe

\begin{tabular}{llccl}
\hline \hline GRB-SN & $\begin{array}{c}M_{\mathrm{Ni}} \\
\left(M_{\odot}\right)\end{array}$ & $\begin{array}{c}E_{K} \\
\left(10^{51} \mathrm{erg}\right)\end{array}$ & $\begin{array}{c}M_{\mathrm{ej}} \\
\left(M_{\odot}\right)\end{array}$ & \multicolumn{1}{c}{ Reference } \\
\hline 1998bw & 0.7 & 30 & 11 & Iwamoto et al. (1998) \\
$2003 \mathrm{dh}$ & 0.35 & 38 & 8 & Mazzali et al. (2003) \\
$20031 \mathrm{w}$ & 0.55 & 60 & 13 & Mazzali et al. (2006b) \\
2006aj & 0.2 & 2 & 2 & Mazzali et al. (2006a) \\
2010bh & 0.1 & 14 & 2.2 & Cano et al. (2011) \\
2009nz (nominal) & 0.35 & 2.3 & 1.4 & This paper \\
2009nz (maximal) & 0.6 & 8.4 & 3.5 & This paper \\
\hline
\end{tabular}

\section{DISCUSSION AND CONCLUSIONS}

We presented a spectrum of the photometric bump (designated SN 2009nz) associated with the standard cosmological GRB 091127, and unambiguously demonstrated that it is a broad-lined SN, similar to the Type Ic SNe associated with nearby GRBs. The best match over the wavelength range of our spectrum $\left(\lambda_{\text {rest }} \approx 4000-6300 \AA\right)$ is provided by SN 2006aj; SNe 1998bw and 2010bh, on the other hand, exhibit broader features indicative of larger expansion velocities. Identifying the absorption feature centered at $6000 \AA$ with Si II $\lambda 6355$, we infer a photospheric velocity about $17,000 \mathrm{~km} \mathrm{~s}^{-1}$ at $\delta t_{\text {rest }} \approx 16.3$ days, similar to that of SN 2006aj, and lower than SNe 1998bw and 2010bh. Combined with the SN light curve properties, we find that the explosion properties of SN $2009 \mathrm{nz}$ are $E_{K, 51} \approx 2.3$, $M_{\mathrm{ej}, \odot} \approx 1.4$, and $M_{\mathrm{Ni}, \odot} \approx 0.35$, similar to those of SN 2006aj.

These results demonstrate that beyond the basic need to spectroscopically confirm photometric bumps as supernovae, spectroscopy of GRB-SNe at $z \gtrsim 0.3$ provides two key measurements. First, the spectra allow us to determine the SN type. To date, all the nearby well-studied GRB-SNe have been classified as broad-lined Type Ic events. At $z \gtrsim 0.3$ there are claims for a normal Type Ic event (GRB 021211/SN 2002lt; Della Valle et al. 2003) and a Type IIn event (GRB 011121/SN 2001ke; Garnavich et al. 2003). However, both of these claims 
are problematic: SN 2002lt equally resembles the peculiar Type Ia SN 1991bg, while SN 2001ke suffers from large, and uncertain, extinction leading to an uncertain spectral shape. In addition, in neither case was the host contribution accounted for, leading to possible contamination of the spectra. The remaining three events at $z \gtrsim 0.3$ with spectroscopic observations (XRF 020903, GRB 050525A, and GRB 101219B) broadly resemble SN 1998bw (Soderberg et al. 2005; Della Valle et al. 2006; Sparre et al. 2011), but no attempt has been made to extract their physical properties. Here, we find that SN 2009nz is also a broad-lined event, but for the first time have a sufficiently large comparison sample to demonstrate a closer similarity to SN 2006aj than to the canonical SN 1998bw.

Second, the spectra allow us to measure the photospheric velocity and, along with the light curve properties, to infer the basic explosion parameters (the light curve by itself only provides a ratio of the kinetic energy and ejecta mass). Thus, spectroscopy provides deeper insight into the physical properties of GRB-SNe than light curves alone. Here, for the first time, we measured the expansion velocity and explosion properties of a GRB-SN at $z \gtrsim 0.2$. Using these measurements we find that SN 2009nz follows the overall trend of lower $M_{\mathrm{Ni}}$ for events with lower $E_{K}$ and $M_{\mathrm{ej}}$ seen in the nearby GRB-SNe (Figure 3).

In the same vein, the similarity in spectral and explosion properties of SN 2009nz and SN 2006aj is instructive in light of the differences between the prompt emission properties of GRB 091127 and XRF 060218. The former is a typical cosmological GRB with $E_{\gamma \text {,iso }} \approx 1.1 \times 10^{52} \mathrm{erg}$, a duration of $T_{90} \approx 9 \mathrm{~s}$, and a rest-frame $E_{p} \approx 25 \mathrm{keV}$ (Wilson-Hodge \& Preece 2009), while the latter is an X-ray flash with $E_{\gamma, \text { iso }} \approx$ $6.2 \times 10^{49} \mathrm{erg}$, a duration of $T_{90} \approx 2100 \mathrm{~s}$, and a rest-frame $E_{p} \approx 5 \mathrm{keV}$ (Campana et al. 2006). These differences provide evidence for a lack of correlation between the prompt emission and associated SN properties. Moreover, it has been suggested that XRF 060218/SN 2006aj was powered by a neutron star instead of a black hole based on the lower ejecta mass and kinetic energy compared to SNe 1998bw, 2003dh, and 20031w, as well as the low energy of the prompt emission (Mazzali et al. 2006a; Soderberg et al. 2006a). However, GRB 091127 is typical of the cosmological GRB population, which is thought to be powered by black holes (MacFadyen \& Woosley 1999), and yet its associated SN 2009nz shares the explosion properties of SN 2006aj. This either casts doubt on the idea that the properties of SN 2006aj (or of GRB-SNe in general) require a neutron star central engine, or alternatively suggests that standard GRBs can be powered by either neutron stars or black holes, but with no clear imprint on the prompt emission properties.

From an observational point of view, we stress that while the broad SN features are identifiable in the bump (supernova+host) spectrum of SN 2009nz, subtraction of the host spectrum provides a more reliable and robust identification of the SN features. Accounting for the host contribution in GRB-SN spectra at $z \gtrsim 0.3$ is critical since in all cases to date the host galaxy contributes about half of the observed flux. ${ }^{14}$ None of the studies published to date accounted for the actual host spectrum, either neglecting its contribution altogether (GRB 021211: Della Valle et al. 2003; GRB 011121: Garnavich et al. 2003), assuming a template blue galaxy spectrum (GRB 050525A: Della Valle et al. 2006; XRF 020903: Soderberg et al. 2005), or assuming a flat spectrum (GRB 101219B: Sparre et al. 2011). In this

14 The afterglow contribution, on the other hand, is generally $\lesssim 10 \%$, and it is also less of a concern due to its featureless spectrum. study we find that either an actual spectrum of the host or an appropriate template matched to the host colors should be subtracted.

We end by noting that the small sample of GRB-SNe with detailed spectroscopic observations already reveals a wide dispersion in explosion properties, with about a factor of 30 in $E_{K}$, a factor of 15 in $M_{\mathrm{ej}}$, and a factor of 7 in $M_{\mathrm{Ni}}$ (Table 2 and Figure 3). This also seems to be the case for the two events associated with standard GRBs-GRB 030329/SN 2003dh and GRB 091127/SN 2009nz. The wide dispersion contradicts earlier claims for a narrow range of GRB-SN properties, mainly their kinetic energies (Kaneko et al. 2007). Clearly, a larger number of events are required to fully sample the range of explosion parameters, to evaluate whether there really exists a dispersion in GRB-SN types, and to investigate any correlations with the GRB properties or differences between the $\mathrm{SNe}$ associated with sub-energetic and standard GRBs. Such a study requires a concerted effort to determine the afterglow properties from broadband modeling, to measure the SN light curves, and to obtain spectra of the bumps and host galaxies. With a Swift detection rate of 2-3 $z \sim 0.5$ GRBs per year such a concerted effort is both desirable and achievable.

E.B. acknowledges partial support from Swift AO6 grant NNX10AI24G. This work is based in part on observations obtained at the Gemini Observatory, which is operated by the Association of Universities for Research in Astronomy, Inc., under a cooperative agreement with the NSF on behalf of the Gemini partnership: the National Science Foundation (United States), the Science and Technology Facilities Council (United Kingdom), the National Research Council (Canada), CONICYT (Chile), the Australian Research Council (Australia), Ministério da Ciência e Tecnologia (Brazil), and Ministerio de Ciencia, Tecnología e Innovación Productiva (Argentina). This paper includes data gathered with the $6.5 \mathrm{~m}$ Magellan Telescopes located at Las Campanas Observatory, Chile.

\section{REFERENCES}

Arnett, W. D. 1982, ApJ, 253, 785

Berger, E., Kulkarni, S. R., Pooley, G., et al. 2003, Nature, 426, 154 Bloom, J. S., Kulkarni, S. R., \& Djorgovski, S. G. 2002, AJ, 123, 1111 Bloom, J. S., Kulkarni, S. R., Djorgovski, S. G., et al. 1999, Nature, 401, 453 Bloom, J. S., Kulkarni, S. R., Price, P. A., et al. 2002, ApJ, 572, L45 Campana, S., Mangano, V., Blustin, A. J., et al. 2006, Nature, 442, 1008 Cano, Z., Bersier, D., Guidorzi, C., et al. 2011, arXiv:1104.5141 Chornock, R., Berger, E., Levesque, E. M., et al. 2010, arXiv:1004.2262 Cobb, B. E., Bloom, J. S., Perley, D. A., et al. 2010, ApJ, 718, L150 Cucchiara, A., Fox, D., Levan, A., \& Tanvir, N. 2009, GCN Circ., 202, 1 Della Valle, M., Malesani, D., Benetti, S., et al. 2003, A\&A, 406, L33 Della Valle, M., Malesani, D., Bloom, J. S., et al. 2006, ApJ, 642, L103 Drout, M. R., Soderberg, A. M., Gal-Yam, A., et al. 2010, arXiv:1011.4959 Esin, A. A., \& Blandford, R. 2000, ApJ, 534, L151

Galama, T. J., Vreeswijk, P. M., van Paradijs, J., et al. 1998, Nature, 395, 670 Garnavich, P. M., Stanek, K. Z., Wyrzykowski, L., et al. 2003, ApJ, 582, 924 Hjorth, J., Sollerman, J., Mller, P., et al. 2003, Nature, 423, 847 Iwamoto, K., Mazzali, P. A., Nomoto, K., et al. 1998, Nature, 395, 672 Kaneko, Y., Ramirez-Ruiz, E., Granot, J., et al. 2007, ApJ, 654, 385 Lazzati, D., Covino, S., Ghisellini, G., et al. 2001, A\&A, 378, 996 MacFadyen, A. I., \& Woosley, S. E. 1999, ApJ, 524, 262 Malesani, D., Tagliaferri, G., Chincarini, G., et al. 2004, ApJ, 609, L5 Matheson, T., Garnavich, P. M., Stanek, K. Z., et al. 2003, ApJ, 599, 394 Mazzali, P. A., Deng, J., Nomoto, K., et al. 2006a, Nature, 442, 1018 Mazzali, P. A., Deng, J., Pian, E., et al. 2006b, ApJ, 645, 1323 Mazzali, P. A., Deng, J., Tominaga, N., et al. 2003, ApJ, 599, L95 Modjaz, M., Stanek, K. Z., Garnavich, P. M., et al. 2006, ApJ, 645, L21 Patat, F., Cappellaro, E., Danziger, J., et al. 2001, ApJ, 555, 900 Pian, E., Mazzali, P. A., Masetti, N., et al. 2006, Nature, 442, 1011 Schlegel, D. J., Finkbeiner, D. P., \& Davis, M. 1998, ApJ, 500, 525 
Soderberg, A. M., Kulkarni, S. R., Berger, E., et al. 2004, Nature, 430, 648 Soderberg, A. M., Kulkarni, S. R., Fox, D. B., et al. 2005, ApJ, 627, 877 Soderberg, A. M., Kulkarni, S. R., Nakar, E., et al. 2006a, Nature, 442, 1014 Soderberg, A. M., Kulkarni, S. R., Price, P. A., et al. 2006b, ApJ, 636, 391 Sparre, M., Sollerman, J., Fynbo, J. P. U., et al. 2011, ApJ, 735, L24
Stanek, K. Z., Matheson, T., Garnavich, P. M., et al. 2003, ApJ, 591, L17 Wainwright, C., Berger, E., \& Penprase, B. E. 2007, ApJ, 657, 367 Waxman, E., \& Draine, B. T. 2000, ApJ, 537, 796

Wilson-Hodge, C. A., \& Preece, R. D. 2009, GCN Circ., 204, 1 Woosley, S. E., \& Bloom, J. S. 2006, ARA\&A, 44, 507 